\title{
PROMPT PLANETESIMAL FORMATION BEYOND THE SNOW LINE
}

\author{
Philip J. Armitage ${ }^{1,2}$, Josh A. Eisner ${ }^{1,3}$, AND Jacob B. Simon ${ }^{4,5}$ \\ ${ }^{1}$ JILA, University of Colorado and NIST, 440 UCB, Boulder, CO 80309-0440, USA; pja@jilau1.colorado.edu \\ ${ }^{2}$ Department of Astrophysical and Planetary Sciences, University of Colorado, Boulder, USA \\ ${ }^{3}$ Steward Observatory, University of Arizona, 933 N. Cherry Avenue, Tucson, AZ 85721-0065, USA \\ ${ }^{4}$ Department of Space Studies, Southwest Research Institute, Boulder, CO 80302, USA \\ Received 2016 May 26; revised 2016 August 1; accepted 2016 August 11; published 2016 August 25
}

\begin{abstract}
We develop a simple model to predict the radial distribution of planetesimal formation. The model is based on the observed growth of dust to millimeter-sized particles, which drift radially, pile-up, and form planetesimals where the stopping time and dust-to-gas ratio intersect the allowed region for streaming instability-induced gravitational collapse. Using an approximate analytic treatment, we first show that drifting particles define a track in metallicitystopping time space whose only substantial dependence is on the disk's angular momentum transport efficiency. Prompt planetesimal formation is feasible for high particle accretion rates (relative to the gas, $\dot{M}_{p} / \dot{M} \gtrsim 3 \times 10^{-2}$ for $\alpha=10^{-2}$ ), which could only be sustained for a limited period of time. If it is possible, it would lead to the deposition of a broad and massive belt of planetesimals with a sharp outer edge. Numerically including turbulent diffusion and vapor condensation processes, we find that a modest enhancement of solids near the snow line occurs for centimeter-sized particles, but that this is largely immaterial for planetesimal formation. We note that radial drift couples planetesimal formation across radii in the disk, and suggest that considerations of planetesimal formation favor a model in which the initial deposition of material for giant planet cores occurs well beyond the snow line.
\end{abstract}

Key words: accretion, accretion disks - instabilities - planets and satellites: formation - protoplanetary disks

\section{INTRODUCTION}

Where and when planetesimals form within protoplanetary disks set the initial conditions for the gravity-dominated phase of planet formation. Meteoritic evidence is consistent with planetesimals forming early, with Schiller et al. (2015) suggesting that differentiated bodies formed only $0.25 \mathrm{Myr}$ after calcium-aluminum-rich inclusions, but little is known empirically about the radial profile beyond the general observation that the solar system, and debris disks, formed planetesimals at a range of radii. One can try to back out the initial distribution of planetesimals from the observed architecture of planetary systems (as in the Minimum Mass Solar Nebula, Hayashi 1981), but this is an ill-posed problem if planets migrate (Kley \& Nelson 2012). Given recent advances in the characterization of gas and dust in protoplanetary disks, a forward-modeling approach that combines observations with planetesimal formation theory may prove at least as instructive.

Here, we develop a simplified global model for where planetesimals form based on known physical processes. Experiments and related modeling suggest that dust grows rapidly up to $\sim$ millimeter sizes, defined by the onset of bouncing (Blum \& Wurm 2008; Zsom et al. 2010). We assume that this remains true beyond the snow line, although the physics of ice coagulation can differ substantially from that of silicates (Dominik \& Tielens 1997; Gundlach \& Blum 2015), in a direction that allows larger particles to form (Wada et al. 2011; Kataoka et al. 2013). The small macroscopic solids that result from coagulation are then subject to radial drift (Weidenschilling 1977) and turbulent diffusion (Clarke \& Pringle 1988), which are sufficiently rapid as to lead to an approximate steady-state on small scales. As the ice-dominated solids cross the snow line they evaporate, and the vapor diffuses outward and recondenses, forming an enhancement of

\footnotetext{
${ }^{5}$ Sagan Fellow.
}

the solid surface density (Stevenson \& Lunine 1988). At radii where the dust-to-gas ratio and dimensionless stopping time fall within certain ranges (determined by Carrera et al. 2015), the streaming instability (Youdin \& Goodman 2005) results in the rapid formation of planetesimals. Planetesimals formed from the streaming instability are characteristically large (Johansen et al. 2007, 2012; Simon et al. 2016), and to a good approximation they will be immune to aerodynamic drift and stay in place.

The above sketch defines a model for "prompt" planetesimal formation that does not invoke helpful but less well-understood processes (such as "lucky growth" beyond material barriers, and local concentration in zonal flows, vortices, or persistent particle traps; for reviews see Johansen et al. 2014; Armitage 2015). It is obviously incomplete. Our intent is to highlight in the simplest possible model the critical role of the competition between radial drift and planetesimal formation.

\section{A MODEL FOR PLANETESIMAL FORMATION FROM DRIFTING SOLIDS}

Consider a gas disk around a star of mass $M_{*}$ that is parameterized by the accretion rate $\dot{M}$, angular momentum transport efficiency parameter $\alpha$ (Shakura \& Sunyaev 1973), and central temperature profile $T(r) \propto r^{-\beta}$, with $\beta$ as a constant. In steady-state,

$$
\nu \Sigma=\frac{\dot{M}}{3 \pi},
$$

where the effective viscosity is given in terms of the sound speed $c_{s}$ and angular velocity $\Omega$ by $\nu=\alpha c_{s}^{2} / \Omega$, and $c_{s}^{2}=k_{\mathrm{B}} T / \mu m_{H}$. Here $k_{\mathrm{B}}$ is the Boltzmann constant, $\mu \simeq 2.2$ is the mean molecular weight, and $m_{H}$ is the mass of a hydrogen atom. The central density of the disk is 
$\rho_{0}=\Sigma / \sqrt{2 \pi} h$, where $\Sigma$ is the surface density and $h=c_{s} / \Omega$ is the vertical scale height.

A constant $\alpha$ model provides a convenient link between $\dot{M}$ and $\Sigma$ using only a single free parameter, though it is unlikely to provide a good representation of protoplanetary disks. Simulations show that the effective $\alpha$ due to magnetohydrodynamic processes varies with radius (Simon et al. 2015), and that the accretion stress is largely divorced from the strength of turbulent diffusion. (In what follows we implicitly assume that turbulent diffusion is weak, allowing particles to settle.) For particle flows, however, what matters most are the profiles of surface density, temperature, and (particularly) pressure. Our fiducial model has $T=150(r / 3 \mathrm{au})^{-0.5} \mathrm{~K}$ and $\Sigma \propto r^{-1}$. The normalized pressure gradient parameter,

$$
\Delta \equiv-\frac{1}{2}\left(\frac{c_{s}}{v_{K}}\right) \frac{d \ln P}{d \ln r},
$$

which determines in part the strength of the streaming instability (Bai \& Stone 2010), has a value $(\Delta \simeq 0.05$ at $1 \mathrm{au})$ that is compatible with the simulations we compare against (Carrera et al. 2015).

To develop an approximate analytic model for the distribution of solids within the disk we note that small macroscopic particles that have grown by coagulation outside the snow line fall in the Epstein drag regime. For particles of material density $\rho_{\mathrm{m}}$ and radius $s$, the dimensionless stopping time is,

$$
\tau_{s}=\frac{\pi}{2} \frac{\rho_{\mathrm{m}}}{\Sigma} s
$$

The aerodynamic drift rate is (Takeuchi \& Lin 2002),

$$
v_{\mathrm{r}}=\frac{\tau_{s}^{-1} v_{\mathrm{r}, \mathrm{gas}}-\eta v_{K}}{\tau_{s}+\tau_{s}^{-1}}
$$

where $v_{\mathrm{r} \text {,gas }}$ is the radial velocity of the gas, $v_{\mathrm{K}}$ is the Keplerian velocity, and $\eta=-(h / r)^{2}(\beta / 2-3)$ is a parameter measuring the degree of radial pressure support for the gas disk. We take the limit where $\tau_{s}$ is small and $\left|v_{\mathrm{r} \text {,gas }}\right| \ll\left|\eta \tau_{s} v_{K}\right|$. For a steadystate radial particle flow, with an accretion rate $\dot{M}_{p}$ and a surface density $\Sigma_{p}$,

$$
\frac{\Sigma_{p}}{\Sigma}=\frac{3 \alpha}{2(3-\beta / 2)} \frac{\dot{M}_{p}}{\dot{M}} \tau_{s}^{-1} .
$$

This expression shows how the solid-phase "metallicity" $Z \equiv \Sigma_{p} / \Sigma$ depends upon the disk properties and the ratio of solid-to-gas accretion rates for given $\tau_{s}$.

Writing the familiar physics of radial drift in the above form is useful because the conditions for the streaming instability to lead to gravitational collapse and planetesimals can also be expressed as an allowed region in $Z-\tau_{s}$ space. A polynomial fit to the simulation results of Carrera et al. (2015) gives the critical metallicity as ${ }^{6}$,

$$
\begin{aligned}
\log Z_{\text {crit }}= & 2.84 \times 10^{-2}\left(\log \tau_{s}\right)^{4}+0.11\left(\log \tau_{s}\right)^{3} \\
& +0.38\left(\log \tau_{s}\right)^{2}+0.6\left(\log \tau_{s}\right)-1.52 .
\end{aligned}
$$

\footnotetext{
6 Carrera et al. (2015) model disks with negligibly weak intrinsic turbulence. Significant levels of turbulence would impede settling and planetesimal formation, especially at low $\tau_{s}$. Our results are thus most applicable to disks in which the accretion stress is primarily laminar, with low levels of turbulence and attendant diffusion.
}

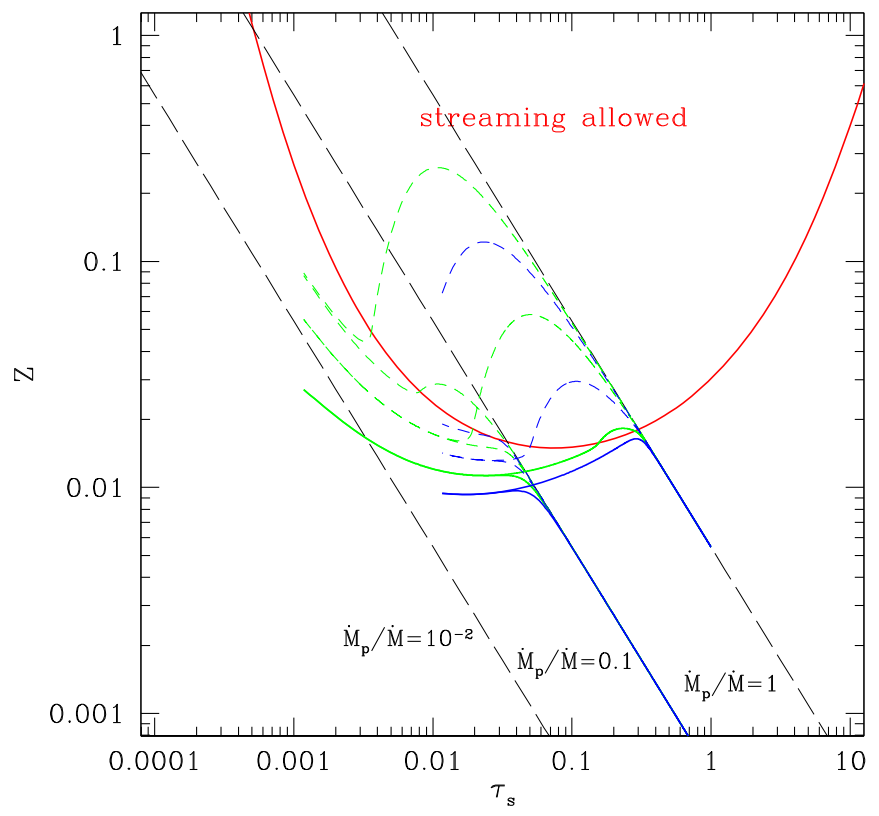

Figure 1. Analytic tracks (dashed lines) in $Z-\tau_{s}$ space for a disk with $\alpha=10^{-2}, \beta=1 / 2$, and various ratios of the particle-to-gas accretion rate. Particles evolve from the lower right to the upper left under radial drift. The red curve defines the approximate region within which prompt planetesimal formation is possible. The green (for millimeter-sized particles) and blue (centimeter-sized) curves show tracks that include a sink term for planetesimal formation, for $K=10^{2}$ (solid curves), $K=10^{3}$ and $K=10^{4}$ (dashed curves). The disk model has $\dot{M}=10^{-8} M_{\odot} \mathrm{yr}^{-1}$ and $T=150(r / 3 \mathrm{au})^{-0.5} \mathrm{~K}$.

Equation (5) then defines a line in that space that either does or does not admit planetesimal formation. Whether planetesimal formation is possible depends on $\alpha$ and the particle flux but does not depend on the actual physical size to which solids grow. Plotting these tracks in Figure 1 for $\alpha=10^{-2}$ (at the high end of observationally estimated values, Hartmann et al. 1998), we see that if $\dot{M}_{p} / \dot{M}=10^{-2}$ the metallicity is too low at any $\tau_{s}$ to allow prompt planetesimal formation. Planetesimal formation is only possible in the outer disk if $\dot{M}_{p} / \dot{M} \gtrsim 3 \times 10^{-2}$, i.e., for relative accretion rates that exceed the fiducial dust-to-gas ratio of $10^{-2}$. This implies that planetesimal formation would occur while the global average of the dust-to-gas ratio was decreasing with time, presumably early on.

A steady-state model will only be valid, even approximately, if particles can grow to a size set by material barriers before they drift significantly (otherwise they will be in the regime of "drift-limited growth," Birnstiel et al. 2012). Birnstiel et al. (2012) estimate that a particle grows from radius $s_{0}$ to $s$ on a timescale,

$$
t_{\text {grow }} \approx \frac{1}{\Omega} \frac{\Sigma}{\Sigma_{p}} \ln \left(\frac{s}{s_{0}}\right)
$$

Adopting $s_{0}=\mu \mathrm{m}, s=\mathrm{mm}$, and $\beta=0.5$, we find that the ratio between the growth time and the drift time $t_{\text {drift }}=r /\left|v_{r}\right|$ is,

$$
\frac{t_{\text {grow }}}{t_{\text {drift }}} \approx 87\left(\frac{\alpha}{10^{-2}}\right)^{-1}\left(\frac{h / r}{0.05}\right)^{2}\left(\frac{\dot{M} / \dot{M}_{p}}{10}\right) \tau_{s}^{2}
$$


For these parameters, the track given by Equation (5) intersects the allowed streaming region at $\tau_{s} \simeq 0.035$, at which point the growth time is a small fraction of the drift time. This ordering is not, however, robust, and for larger $\tau_{s}$ growth may be slower than drift.

To model the impact of planetesimal formation on the track in $Z-\tau_{s}$ space, we integrate the continuity equation for the particles,

$$
\frac{\partial \Sigma_{p}}{\partial t}+\frac{1}{r} \frac{\partial}{\partial r}\left(r \Sigma_{p} v_{r}\right)=-\frac{\Sigma_{p}}{t_{\text {form }}}
$$

to find a steady-state solution. We assume that planetesimals form on a multiple $K$ of the dynamical timescale within the allowed region, $t_{\text {form }}=K \Omega^{-1}$. For $Z<Z_{\text {crit }}$ we smoothly suppress the rate by a factor $\exp \left[\left(Z_{\text {crit }}-Z\right) / 0.1 Z_{\text {crit }}\right]$. This roll-off in the planetesimal formation rate is imposed for numerical convenience, though it is physically plausible that some planetesimal formation persists at metallicities just below the nominal threshold. We consider icy particles $\left(\rho_{m}=1 \mathrm{~g} \mathrm{~cm}^{-3}\right)$ in a disk with $\dot{M}=10^{-8} M_{\odot} \mathrm{yr}^{-1}$, $\alpha=10^{-2}$, and $T=150(r / 3 \mathrm{au})^{-0.5} \mathrm{~K}$.

Figure 1 shows solutions to this model. The key point is that, within the allowed region, the timescale for prompt planetesimal formation scales with radius as $r^{-3 / 2}$, which can be compared to the timescale for radial drift $t_{\text {drift }}=$ const. There is therefore a critical radius within which planetesimal formation dominates, whereas outside radial drift leads to particle pile-up (Youdin \& Chiang 2004). If the streaming instability leads to gravitational collapse on an essentially dynamical timescale ( $K \sim 10^{2}$, as is found in simulations, e.g., Simon et al. 2016), then the derived $Z-\tau_{s}$ tracks skirt the lower boundary of the allowed region. Slower planetesimal formation timescales lead to clearly defined regions where first radial drift and then planetesimal formation dominate. We remark that models with large values of $K$ have an alternate interpretation in terms of stochastic planetesimal formation. For example, a model with $\dot{M}_{p} / \dot{M}=1$ and $K=10^{4}$ is equivalent to one with $\dot{M}_{p} / \dot{M}=10^{-2}$ if transient local concentrations attain the metallicity needed for prompt planetesimal formation (with $\left.K=10^{2}\right) 1 \%$ of the time.

Figure 2 shows the solutions as a function of radius (notionally extended into $1 \mathrm{au}$, though this would be inward of the physical snow line). For $\dot{M}_{p} / \dot{M} \gtrsim 0.1$ and millimetersized to centimeter-sized particles, planetesimal formation in the outer disk is an efficient sink for the radial flow of solids. The mass accretion rate of solids reaching the snow line is substantially reduced, by a factor that ranges from several to more than an order of magnitude. As a result, the rate of planetesimal formation at any given radius depends on whether planetesimals have formed further out in the disk. For most of the models considered the sweet spot for planetesimal formation is well outside the snow line, in some cases as far out as 30-40 au. Planetesimals are predicted to be laid down across a broad range of radii for high particle fluxes, whereas lower fluxes yield a narrower distribution. The outer cut-off will be sharp if, as simulations suggest (Johansen et al. 2009), planetesimal formation becomes rapidly inefficient below a threshold metallicity.

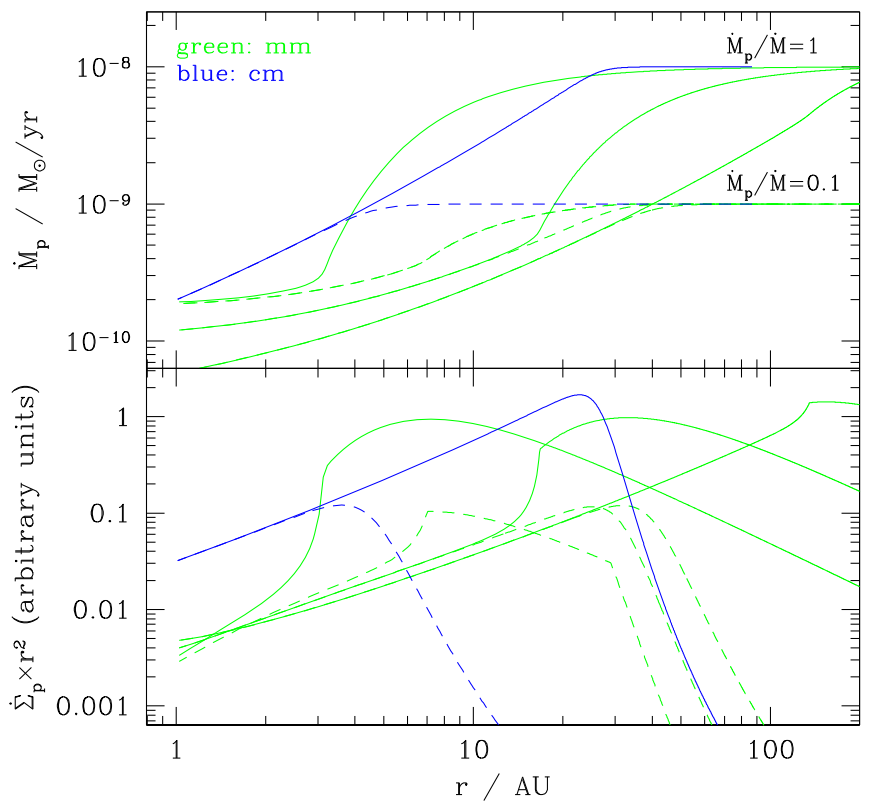

Figure 2. Radial projection of the models shown in Figure 1. Upper panel: the radial dependence of the particle mass accretion rate. Lower panel: the radial dependence of planetesimal formation. Solid curves refer to models with $\dot{M}_{p} / \dot{M}=1$, and dashed curves refer to models with $\dot{M}_{p} / \dot{M}=0.1$. The assumed planetesimal formation time for the millimeter-sized particles cases increases from right to left $\left(K=10^{2}, 10^{3}, 10^{4}\right)$. For centimeter-sized particles only the case with $K=10^{2}$ is plotted.

\section{PARTICLE AND VAPOR DIFFUSION}

We now explore how robust these conclusions are to neglected physical effects. Turbulent particle diffusion works against particle pile-up (Hughes \& Armitage 2012), further favoring large radii as sites for planetesimal formation, but the condensation of vapor diffusing across the snow line has the opposite effect (Stevenson \& Lunine 1988). We adopt a timedependent treatment (Alexander \& Armitage 2007) and solve continuity equations that treat solids and vapor as trace species, with surface density $\Sigma_{t}$,

$$
\frac{\partial \Sigma_{t}}{\partial t}+\frac{1}{r} \frac{\partial}{\partial r}\left[r\left(F_{\text {diff }}+\Sigma_{t} v_{r}\right)\right]=0 .
$$

The diffusive term $F_{\text {diff }}=-D \Sigma \partial\left(\Sigma_{t} / \Sigma\right) / \partial r$ (Clarke \& Pringle 1988), where $D$ is the diffusion co-efficient (here set equal to $\nu$ for both particles and vapor), and $v_{r}$ is the gas radial velocity (for the vapor) or the aerodynamic drift speed (for particles). We supplement these equations with source / sink terms that are appropriate for instantaneous sublimation and condensation of water vapor, following the method and chemical constants given in Ciesla \& Cuzzi (2006). In brief, at radii where ice is not fully sublimated, we sublimate or condense the appropriate amount of ice or vapor at each time step to maintain the actual vapor pressure of water at the equilibrium value given by the Clausius-Clapeyron equation. We assume that there is no significant change to the size distribution of particles in the vicinity of the snow line, an assumption that minimizes its importance. In more complete models, at least some vapor condenses on to pre-existing particles, growing them to larger sizes (Ros \& Johansen 2013).

Figure 3 shows the resulting steady-state profiles of vapor and solids, computed in the fiducial disk model. The solid 


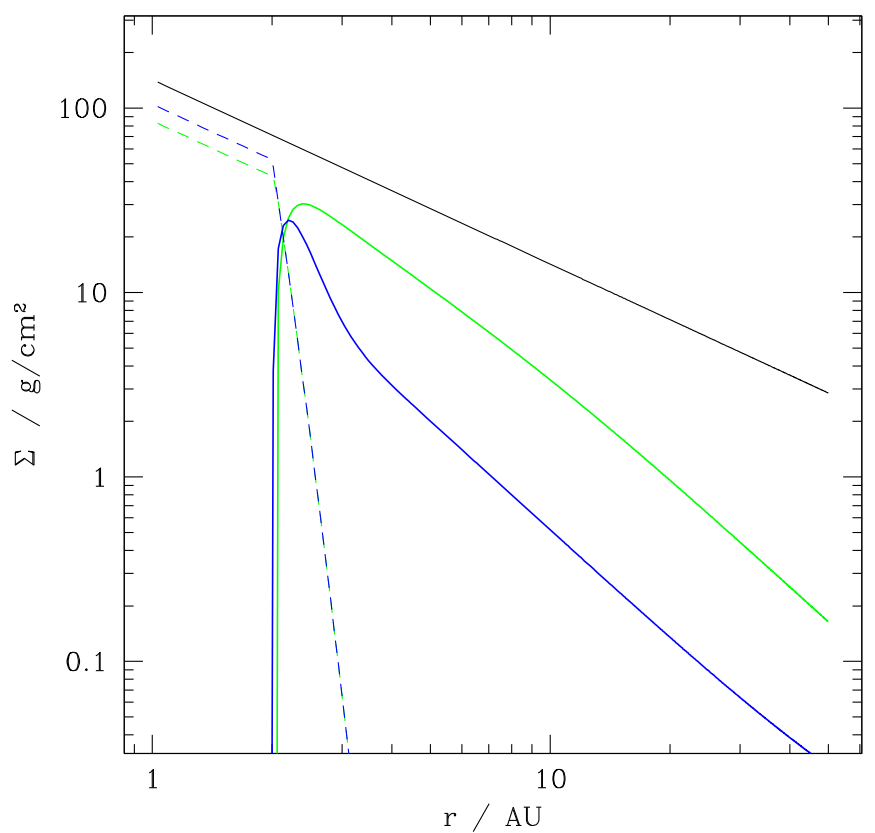

Figure 3. Example steady-state surface density profiles of millimeter-sized (green) and centimeter-sized (blue) particles, water vapor (dashed lines), and gas, for the disk model with $\dot{M}=10^{-8} M_{\odot} \mathrm{yr}^{-1}$. Outward diffusion and condensation of vapor lead to a modest enhancement of the solid surface density outside the snow line for centimeter-sized (and larger) particles.

metallicity (which is freely scalable in models that ignore planetesimal formation) is set to match the analytic results for $\dot{M}_{p} / \dot{M}=1$ at 50 au. For $r \gtrsim 5$ au the surface density of solids is close to a power law, consistent with turbulent diffusion being a minor effect on these scales. For millimeter-sized particles the condensation of diffusing vapor also makes a negligible change to the equilibrium distribution of solids, but for centimeter-sized (and larger) solids a modest enhancement of solids upstream of the snow line occurs. Slightly stronger effects are possible if $D / \nu>1$. Because the radial drift velocity exceeds the gas velocity, the abundance of water vapor interior to the snow line is enhanced as long as large masses of small solids remain present in the disk.

Adopting the same "scale-free" model for local planetesimal formation losses as in Section 2, in Figure 4 we show an illustrative example of how the results shown in Figure 1 are modified by turbulent diffusion and vapor condensation. Just as before, the gas disk has $\dot{M}=10^{-8} M_{\odot} \mathrm{yr}^{-1}$ and $T=150(r / 3 \mathrm{au})^{-0.5} \mathrm{~K}$, which places the snow line at radii similar to those inferred for the solar system (Morbidelli et al. 2000). As was already obvious from Figure 3, for millimeter-sized particles the extra physics in the numerical model makes very little difference to the predicted radii where planetesimals could form. For centimeter-sized particles there is a more significant deviation from the analytic model results. The higher surface density caused by the condensation of diffusing water vapor results in a secondary peak of planetesimal formation just outside the snow line, but does not alter the conclusion that the most-favored location lies further out. This result would be reinforced at higher gas accretion rates, arguably more appropriate to an early phase of disk evolution, which would boost the threshold size for vapor condensation effects to matter.

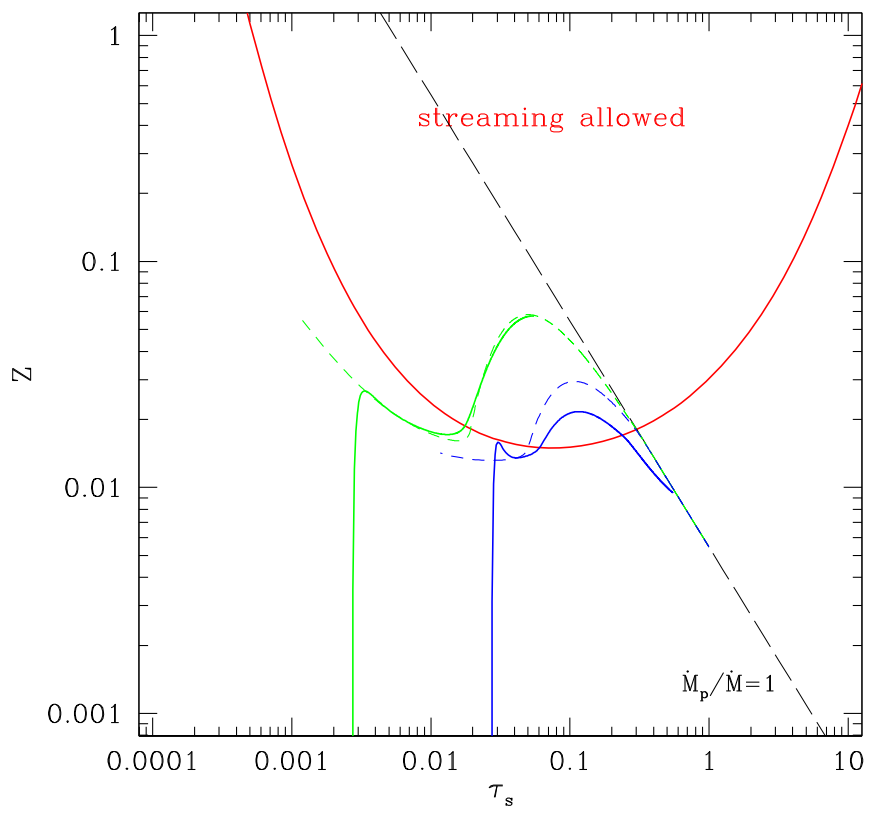

Figure 4. Steady-state tracks for millimeter-sized (green curves) and centimeter-sized (blue) particles in $Z-\tau_{s}$ space, including the effects of turbulent particle diffusion and vapor diffusion / condensation. We assume $\dot{M}_{p} / \dot{M}=1, K=10^{3}$, and the fiducial disk model. The simpler models shown in Figure 1 are plotted as the dashed lines.

\section{DISCUSSION}

At radii beyond the water snow line there is a limited window into prompt planetesimal formation that invokes only known physical processes: coagulation to a fixed size that is of the order of millimeters, radial drift, and gravitational collapse of streaming-initiated overdensities. Planetesimal formation via this route is possible early on-while radial drift is rapidly reducing the global dust-to-gas ratio-and would typically lead to the deposition of a broad and massive belt of planetesimals well outside the snow line. We have not attempted to fine tune the model, but Figure 2 makes it clear that features such as the outer edge of the Kuiper Belt (Trujillo \& Brown 2001) and the large masses of primordial debris required in the Nice model (Tsiganis et al. 2005) are qualitatively consistent with planetesimal formation expectations, as noted by Youdin \& Shu (2002).

Efficient early planetesimal formation is a prerequisite for early planet formation, which has been proposed as an explanation for the ring-like structures seen in ALMA observations of HL Tau (ALMA Partnership et al. 2015). There is tension, however, between other observations and any model that invokes radial drift and pile-up as key ingredients of planetesimal formation. Such models predict a rapid loss of small solids via radial drift and planetesimal formation, whereas observations of $\mathrm{CO}$ line emission toward mostly older sources favor dust-to-gas ratios in excess of $10^{-2}$ (Williams \& Best 2014; Eisner et al. 2016). A better understanding of gas disk mass estimates, which for now differ substantially depending on the technique used (e.g., Bergin et al. 2013; Manara et al. 2016), is the single most important advance that would constrain theoretical models of radial drift.

Our model is deliberately simple, and is intended to provide insight into the physical origins and robustness of more complex approaches. It could be improved by better delineating the conditions needed for planetesimal formation (e.g., by 
including the dependence on the radial pressure gradient, Bai \& Stone 2010), and by modeling the coupled growth and drift of particles. Several authors have already developed such coupled models. Drążkowska \& Dullemond (2014), using a Monte Carlo dust coagulation code, argued for preferential triggering of the streaming instability beyond the ice line. Similarly, Krijt et al. (2016), using a detailed model for particle growth coupled to estimates of planetesimal formation thresholds similar to ours, found that dust-rich disks with weak turbulence promote prompt outer disk planetesimal formation. In the inner disk, there is general agreement that planetesimal formation is harder, and a prompt route is only possible if particles grow to substantially larger sizes (limited only by fragmentation and radial drift, rather than bouncing; Drazkowska et al. 2016). Alternatively, planetesimal formation in the terrestrial planet region may occur via a less-efficient stochastic channel (driven by local turbulent enhancements in $Z$, Johansen et al. 2014), and at still smaller radii at persistent traps associated with the inner edge of the dead zone (Lyra et al. 2009). Despite their differences all models agree that attaining the conditions needed for planetesimal formation is relatively hard, and that as a result the initial distribution of planetesimals is unlikely to be a simple power law but rather a complex function of radius.

Building the cores of the giant planets requires, first, the deposition of a large mass of solids into planetesimals (Pollack et al. 1996). The preference of the streaming instability for relatively large stopping times challenges the common view that the favored location for this deposition is adjacent to the snow line. Even when the effects of vapor diffusion and condensation are considered, we find that the best time and place to lay down a large mass of planetesimals is early and at large radii. This suggests a model for core formation in which planetesimal formation and initial growth occur well beyond the snow line. Subsequent growth could either occur in situ via pebble accretion (Lambrechts \& Johansen 2014; Levison et al. 2015), or via migration to a trap closer in.

P.J.A. thanks Cathie Clarke and the Institute of Astronomy, Cambridge, for hospitality, and acknowledges support from NASA through grants NNX13AI58G and NNX16AB42G, and from NSF AAG grant AST 1313021. J.A.E. acknowledges support from NSF AAG grant 1211329. J.B.S.'s support was provided in part under contract with the California Institute of Technology (Caltech) and the Jet Propulsion Laboratory (JPL), funded by NASA through the Sagan Fellowship Program executed by the NASA Exoplanet Science Institute.

\section{REFERENCES}

Alexander, R. D., \& Armitage, P. J. 2007, MNRAS, 375, 500

ALMA Partnership, Brogan, C. L., Pérez, L. M., et al. 2015, ApJL, 808, L3 Armitage, P. J. 2015, arXiv:1509.06382

Bai, X.-N., \& Stone, J. M. 2010, ApJL, 722, L220

Bergin, E. A., Cleeves, L. I., Gorti, U., et al. 2013, Natur, 493, 644

Birnstiel, T., Klahr, H., \& Ercolano, B. 2012, A\&A, 539, A148

Blum, J., \& Wurm, G. 2008, ARA\&A, 46, 21

Carrera, D., Johansen, A., \& Davies, M. B. 2015, A\&A, 579, A43

Ciesla, F. J., \& Cuzzi, J. N. 2006, Icar, 181, 178

Clarke, C. J., \& Pringle, J. E. 1988, MNRAS, 235, 365

Dominik, C., \& Tielens, A. G. G. M. 1997, ApJ, 480, 647

Drazkowska, J., Alibert, Y., \& Moore, B. 2016, A\&A, in press (arXiv:1607. 05734)

Drążkowska, J., \& Dullemond, C. P. 2014, A\&A, 572, A78

Eisner, J. A., Bally, J. M., Ginsburg, A., \& Sheehan, P. D. 2016, ApJ, 826, 16 Gundlach, B., \& Blum, J. 2015, ApJ, 798, 34

Hartmann, L., Calvet, N., Gullbring, E., \& D'Alessio, P. 1998, ApJ, 495, 385

Hayashi, C. 1981, PThPS, 70, 35

Hughes, A. L. H., \& Armitage, P. J. 2012, MNRAS, 423, 389

Johansen, A., Blum, J., Tanaka, H., et al. 2014, in Protostars and Planets VI, ed. H. Beuther et al. (Tucson, AZ: Univ. Arizona Press), 547

Johansen, A., Oishi, J. S., Mac Low, M.-M., et al. 2007, Natur, 448, 1022

Johansen, A., Youdin, A., \& Mac Low, M.-M. 2009, ApJL, 704, L75

Johansen, A., Youdin, A. N., \& Lithwick, Y. 2012, A\&A, 537, A125

Kataoka, A., Tanaka, H., Okuzumi, S., \& Wada, K. 2013, A\&A, 557, L4

Kley, W., \& Nelson, R. P. 2012, ARA\&A, 50, 211

Krijt, S., Ormel, C. W., Dominik, C., \& Tielens, A. G. G. M. 2016, A\&A, 586, A20

Lambrechts, M., \& Johansen, A. 2014, A\&A, 572, A107

Levison, H. F., Kretke, K. A., \& Duncan, M. J. 2015, Natur, 524, 322

Lyra, W., Johansen, A., Zsom, A., Klahr, H., \& Piskunov, N. 2009, A\&A, 497,869

Manara, C. F., Rosotti, G., Testi, L., et al. 2016, A\&A, 591, L3

Morbidelli, A., Chambers, J., Lunine, J. I., et al. 2000, M\&PS, 35, 1309

Pollack, J. B., Hubickyj, O., Bodenheimer, P., et al. 1996, Icar, 124, 62

Ros, K., \& Johansen, A. 2013, A\&A, 552, A137

Schiller, M., Connelly, J. N., Glad, A. C., Mikouchi, T., \& Bizzarro, M. 2015, E\&PSL, 420, 45

Shakura, N. I., \& Sunyaev, R. A. 1973, A\&A, 24, 337

Simon, J. B., Armitage, P. J., Li, R., \& Youdin, A. N. 2016, ApJ, 822, 55

Simon, J. B., Lesur, G., Kunz, M. W., \& Armitage, P. J. 2015, MNRAS, 454,1117

Stevenson, D. J., \& Lunine, J. I. 1988, Icar, 75, 146

Takeuchi, T., \& Lin, D. N. C. 2002, ApJ, 581, 1344

Trujillo, C. A., \& Brown, M. E. 2001, ApJL, 554, L95

Tsiganis, K., Gomes, R., Morbidelli, A., \& Levison, H. F. 2005, Natur, 435,459

Wada, K., Tanaka, H., Suyama, T., Kimura, H., \& Yamamoto, T. 2011, ApJ, 737,36

Weidenschilling, S. J. 1977, MNRAS, 180, 57

Williams, J. P., \& Best, W. M. J. 2014, ApJ, 788, 59

Youdin, A. N., \& Chiang, E. I. 2004, ApJ, 601, 1109

Youdin, A. N., \& Goodman, J. 2005, ApJ, 620, 459

Youdin, A. N., \& Shu, F. H. 2002, ApJ, 580, 494

Zsom, A., Ormel, C. W., Güttler, C., Blum, J., \& Dullemond, C. P. 2010, A\&A, 513, A57 